\title{
Phenomenon of maximum and minimum in the resistivity of Cu-Mn Kondo alloys at low temperatures
}

\author{
Muhammad A. AL-Jalali \\ Physics Department, Faculty of Science, Taif University, 21974 Taif, P. O. Box 888, kingdom of Saudi Arabia
}

\section{Email address:}

maljalali@yahoo.com

\section{To cite this article:}

Muhammad A. AL-Jalali. Phenomenon of Maximum and Minimum in the Resistivity of Cu-Mn Kondo Alloys at Low Temperatures. American Journal of Physics and Applications. Vol. 2, No. 3, 2014, pp. 78-82. doi: 10.11648/j.ajpa.20140203.12

\begin{abstract}
A New analysis of the electrical resistivity has been studied for $\mathrm{Cu}-\mathrm{Mn}$ Kondo alloys, and it was found a solution like a logarithm power series between resistivity and temperature, which cover all ranges of maximum and minimum in the resistivity and all kinds of interactions at low temperatures. The s-d exchange integral $J_{\text {sd }}$ acting the main role in Kondo effect Phenomenon, $\mathrm{J}_{\mathrm{sd}}$ is negative or positive, according to a kind of interaction between conduction electron spins of the host normal metal and localized spins of the transition element. PACS: 75: 50, 75:10, 75: 40, 75: 30.
\end{abstract}

Keywords: Kondo Effect, Resistivity Maximum, Resistivity Minimum, s-d Interaction

\section{Introduction}

Experimentally, a resistance minimum discovered in the middle of the 1930's in Cu-Fe alloy [1]. Moreover, after that several experiments have confirmed this result $[2,3,4]$

After about 30 years, theoretically, the problem of the resistivity minimum in dilute magnetic alloys was started and discovered by Kondo in 1964[5], and followed by many papers $[6,7,8]$. Since then, Kondo himself and others have put many efforts to developing a complete theory of the so-called Kondo effect [9]. At the same time, with Kondo, many theoretical models discovered to explain this mystery phenomenon [10-21], but until now, the best model is the s-d exchange model, which belong to the first half of RKKY interactions [22,33].

\section{Theoretical Background}

Generally, the total resistivity in dilute magnetic alloys is the sum of many terms, which may include magnetic, phonon, electron- electron scattering, and residual resistivity.

The well-known formula for the total resistivity of dilute magnetic alloys is Kondo's [5]:

$$
\rho=\rho_{\text {phonon }}+c \rho_{A}+c \rho_{M}+c\left(3 z J_{s d} \rho_{M} / E_{F}\right) \operatorname{Ln}(T)
$$

Where $\rho_{\text {phonon }}$ is the phonon resistivity, c is the impurity concentration, $c \rho_{A}$ is the resistivity due to the impurity potential, $c \rho_{M}$ the spin scattering resistivity, and the residual resistivity $\rho_{0}$ is the sum of $\rho_{A}$ and $\rho_{M},\left(E_{F}=\right.$ the Fermi energy), $(Z=$ number of conduction electrons per atom), and Kondo neglect all the terms of the order $\left(\mathrm{KT} / \mathrm{E}_{\mathrm{F}}\right)^{2}$.

It may notes that, with a negative $\mathrm{J}_{\mathrm{sd}}$, the interaction leads to increase localized spin disorder resistivity with decreasing temperature, which may give the following form:

$$
\rho_{\text {spin }}=c \rho_{M}-c\left(3 z J_{s d} \rho_{M} / E_{F}\right) \operatorname{Ln}(T)
$$

In addition, Hamann derived another well-known formula for the magnetic resistivity [23], which has the form:

$$
\begin{aligned}
& \frac{\Delta \rho(T)}{C}=[\Delta \rho(0) / 2 C]\left\{1 \pm \operatorname{Ln}\left(T / T_{k}\right) /\left[\operatorname{Ln}^{2}\left(T / T_{k}\right)+\left(\pi^{2} s(s+1)\right)\right]^{\frac{1}{2}}\right\} \\
& \text { where } \quad+\operatorname{sign} \rightarrow T<T_{k} \quad \text { and } \quad-\operatorname{sign} \rightarrow T>T_{k}
\end{aligned}
$$

Where $\mathrm{T}_{\mathrm{K}}$ is the Kondo's temperature and $\mathrm{S}$ is the spin of the magnetic impurity.

The above expression (3) is in fair agreement with experiments at temperatures $\mathrm{T}>\mathrm{T}_{\mathrm{K}}$ but this agreement breaks down progressively with decreasing temperatures.

In 1986 Zhu et.al.[24] derived a method by application new transport equation to calculate the magnetic resistivity of dilute magnetic alloys. They deduced a resistivity 
expression as a power series in temperature of form:

$$
\rho=\frac{1}{c}\left[1-\frac{x}{c \alpha}+\left(\frac{x}{c \alpha}\right)^{2}-\ldots \ldots \ldots . .\right]
$$

Where $\alpha=1-z \operatorname{Ln}(k T / D)$, and $\mathrm{x}, \mathrm{c}, \mathrm{D}$ some variables connected with s-d exchange interaction and Kondo's temperature, and $\mathrm{z}$ a positive constant when $\mathrm{J}_{\text {sd }}<0$.

The relation (4) does not diverge logarithmically and overcomes the defect of the divergence of the Kondo resistivity at $\mathrm{T}=0$, and has a similar form to Hamman's expression, but not identical with it.

Many recent papers have issued [25, 26, 27, 28, 29, 30] but most of them orbit around the interpretation of the previous articles.

All expressions above were interesting with resistivity minimum and Kondo's temperature, but there is no enough evidence about the maximum in resistivity.

\section{Experimental Background}

Many crude data were reported [2, 3, 5, 9] on electrical resistivity. They come from direct measurements already carried on $\mathrm{Cu}-\mathrm{Mn}$ alloys, and many other noble transition metals alloys. Mn concentrations ranging from $10.5 \mathrm{ppm}$ to $2081.3 \mathrm{ppm}$ in $\mathrm{Cu}$, and temperatures within the range of $(0.03-4.2 \mathrm{~K})$.

In the next section, and on the light of above theoretical background, the experimental data were analyzed above and below Kondo temperature to examine those theoretical relations.

\section{Results and Discussions}

By analysis of experimental data, figure (1) shows $\Delta \rho / c$ versus $\mathrm{Ln}(\mathrm{T} / \mathrm{c})$, where bold lines represent pure magnetic resistivity $\Delta \rho$ as a function of (LnT), and those lines subject to the following relationship.

$$
\begin{aligned}
& \Delta \rho=a-b \operatorname{Ln} T \quad 10^{-8} \Omega . m \\
& \text { When } \quad C=0.0908 \% M n \\
& \Delta \rho / C=a+b \ln (T / C) \\
& r^{2}=0.99984826 \quad a=0.091139764 \quad b=-0.0046047495
\end{aligned}
$$

We could find that, relation (5) matches with Hamann's expression (3).

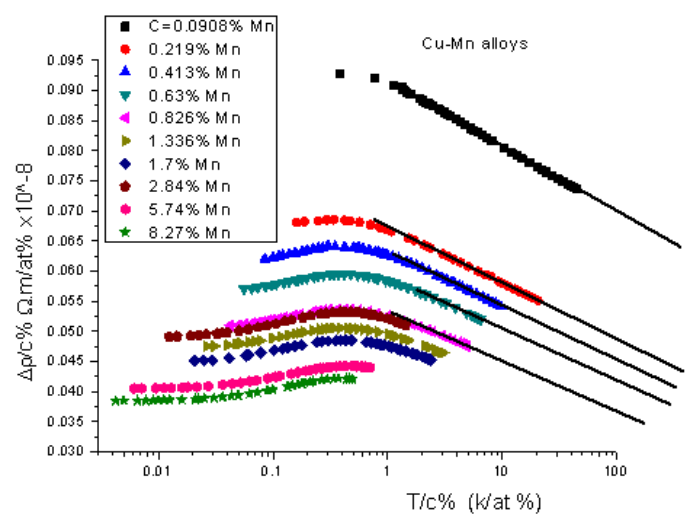

Fig (1). $\Delta \rho / c \% v s$. Ln (T/c\%) for Cu-Mn alloys

A new examine to experimental data has been made by making a suit fitting in the whole temperature range from $\mathrm{T}>>\mathrm{T}_{\mathrm{K}}$ to $\mathrm{T}<<\mathrm{T}_{\mathrm{K}}$. Where in the absence of minimum and maximum experimental data, an appropriate software has made a suitable external extrapolation to show an expecting minimum and maximum in the resistivity. Figures $(2,3,4$, $5,6)$ show a new analysis and give a new logarithm power series solution as this relation:

$$
\begin{aligned}
& \Delta \rho / c=a+b \ln (T / c)+c(\ln T / c)^{2}+d(\ln T / c)^{3}+e(\ln T / c)^{4}+f(\ln T / c)^{5}+g(\ln T / c)^{6}+h(\ln T / c)^{7}+ \\
& i(\ln T / c)^{8}+\mathrm{j}(\ln T / c)^{9}+\mathrm{k}(\ln T / c)^{10}+\ldots . .+n(\ln T / c)^{n}=\sum_{n=0}^{n} a_{n}(\ln T / c)^{n}
\end{aligned}
$$
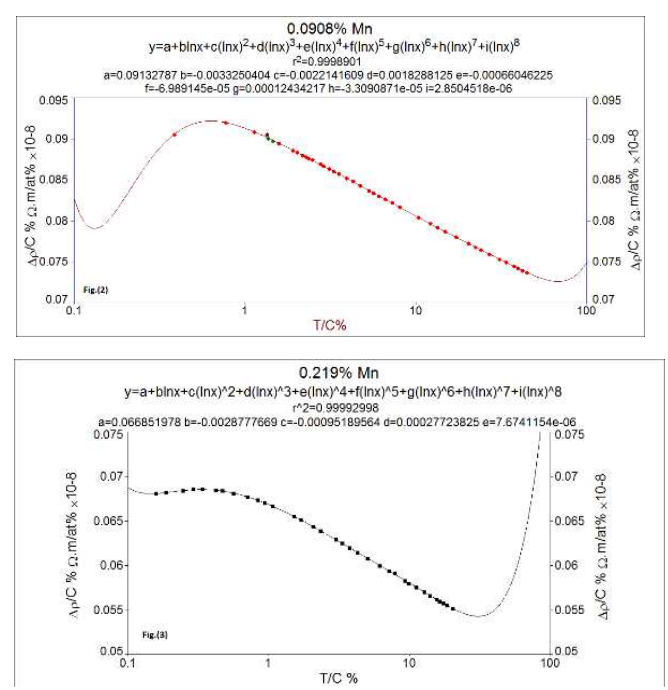
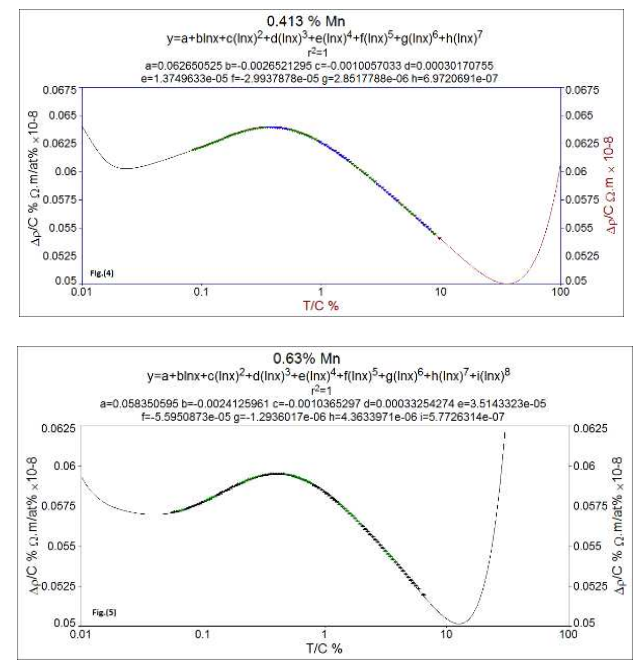


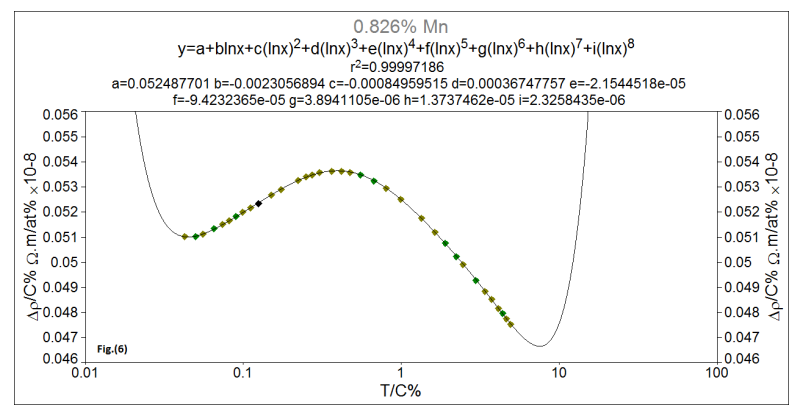

Fig $(2,3,4,5,6) . \Delta \rho / C \%$ vs. Ln (T/c \%) for Cu-Mn alloys.

Expression (6) has all kind of magnetic interaction mechanisms and matches with all theoretical expressions, where, for example, the first two terms consistent with Kondo effect and the task of the theoretical physics is to examine every term in this relation. Figure $(5,6)$ show tendency to spin glass regime, which needs widely investigations.

Values of $\mathrm{J}_{\text {sd }}$ were calculated $[31,32]$ according to a width of internal field distribution at $\mathrm{T}_{\max }$ (characterize the maximum value of the resistivity in figure (1)). Whereas figure (7) represents $T_{\max }$ variation with Mn's concentration and a variation of $\mathrm{J}_{\mathrm{sd}}$ with concentration reveals an expression as the following:

$$
\mathrm{Jsd}=-0.014898811 \operatorname{Ln}(\mathrm{C})-0.17956871 \mathrm{ev}
$$

$\mathrm{C}$ is Mn's concentration in ppm units.

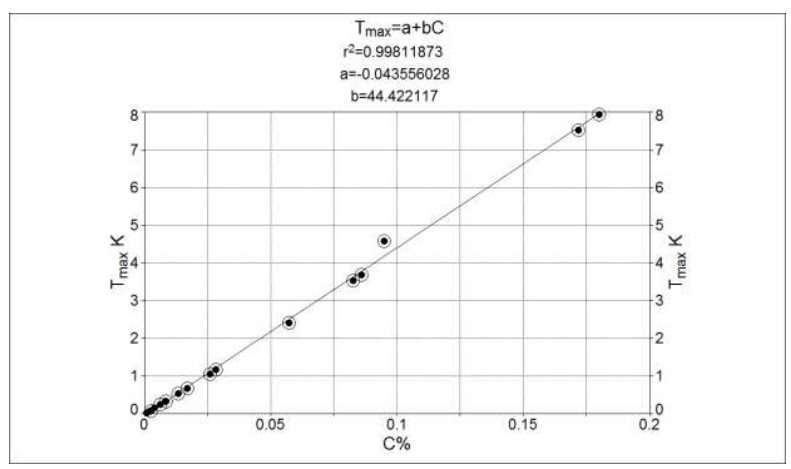

Fig (7). $T_{\max } v s . C \%$ Mn's concentrations.

On the other hand, expression (7) reveals that, Kondo temperature $\left(T_{k}\right)$ which has the following form [25]:

$$
\mathrm{T}_{\mathrm{K}}=\mathrm{T}_{\mathrm{F}} \mathrm{e}^{-\left(\mathrm{n}\left(\mathrm{E}_{\mathrm{F}}\right) \mathrm{J}_{\mathrm{sd}}\right)^{-1}}
$$

$\left(\mathrm{T}_{\mathrm{F}}(\right.$ Fermi temperature $)=8.1210^{4} \mathrm{k}$, and $\left.\mathrm{n}\left(\mathrm{E}_{\mathrm{F}}\right)=0.294 \mathrm{ev}^{-1}\right)$ is strongly variation with changes of concentration, that mean $T_{k}$ is not constant, and not consistent with [33-37].

\section{Conclusion}

In the light of theoretical and experimental achievements, mechanisms of resistivity in magnetic dilute alloys at low temperatures can be distributed to the following regions:

\subsection{Region of Phonons}

At high temperatures, which mean above resistivity minimum, the resistivity of phonons will prevail in spite of many neglecting mechanisms like electron - electron scattering, residual resistivity, and traces of magnetic impurities.

\subsection{Region of Resistivity Minimum}

In this region a conflict between phonons and magnetic impurity resistivity will happen, which yields a resistivity minimum, where mathematically the slope of resistivity will be zero and $\frac{d^{2} \rho}{d T^{2}}>0$.

\subsection{Region between Minimum and Kondo Temperature}

A kind of antiferromagnetic interactions causes a logarithmic temperature dependence versus impurity resistivity, which called Kondo law where $\mathrm{J}_{\mathrm{sd}}<0$, with lowering temperature, Kondo law will vanish at a characteristic lower temperature called Kondo temperature.

\subsection{Region of Maximum in the Resistivity}

The resistivity maximum arises from a combined effect of the Kondo Ln (T) term with suppression of s-d scattering by the ordering of localized Mn moments. And below the Kondo temperature a conflict between antiferromagnetic and ferromagnetic interactions cause a resistivity maximum at a temperature called maximum temperature $\left(\mathrm{T}_{\max }\right)$ is associated with the disappearance of the effective moment of the impurity and $\frac{d^{2} \rho}{d T^{2}}<0$.

Figure (7)shows that $T_{\max }$ is increasing linearly with concentration, which mean that regions of maximum will be more flattened with increasing impurity concentrations, and may be tended to spin glass regime.

\subsection{Region between Resistivity Maximum and Zero Temperature}

At a temperature $\mathrm{T}<\mathrm{T}_{\max }$ a kind of ferromagnetic interactions will happen and a coupling constant will be positive $\mathrm{J}_{\mathrm{sd}}>0$, but at $\mathrm{T}<<\mathrm{T}_{\text {max }}$ the strong coupling between the impurity and the conduction electrons leads to properties drastically different from those observed under and around the maximum temperature. At $\mathrm{T}=0$ there is no completing evidence on this point, but may be a residual resistivity plays a great role in this region.

Finally, the experiments, which had been analyzed in the light of available theoretical results, still closer to the ambiguity, and need more extensive theoretical explanations.

\section{Acknowledgement}

I am greatly indebted to all those whom I analyzed their published crude experimental results. 


\section{References}

[1] W. Meissner, and G. Voigt,"Messungen mit Hilfe von flüssigem Helium XI Widerstand der reinen Metalle in tiefen Temperaturen" Ann. Phys. Volume 399, Issue 7, pages 761797.(1930). DOI: 10.1002/andp.19303990702

[2] S Jacobs. And R. W. Schmitt, " Low-Temperature Electrical and Magnetic Behavior of Dilute Alloys $\mathrm{Mn}$ in $\mathrm{Cu}$ and $\mathrm{Co}$ in $\mathrm{Cu}$,"Phys. Rev.Volume113,Issue 2,Pages: 459-463, (1959). DOI:10.1103/PhysRev.113.459

[3] P. Monod, "Magnetic Field Dependence of the Kondo Resistivity Minimum in CuFeand CuMu Alloys."Phys. Rev. Lett. vol. 19, no. 19, pp. 1113-1117, (1967). DOI: http://dx.doi.org/10.1103/PhysRevLett.19.1113

[4] W. B. Pearson, "Electron Transport in Copper and Dilute Alloys at Low Temperature. IV: Resistance Minimum: Temperature of Occurrence as a Function ofSolute Concentration." Phil. Mag.vol. 46, no. 379, pp. 920-923, (1955). DOI:10.1080/14786440808561245

[5] J. Kondo, "Resistance Minimum in Dilute Magnetic Alloys."Prog.Theor.Phys.3237-49,(1964). DOI:10.1143/PTP.32.37

[6] J. Kondo,"s-d Scattering at Low Temperatures." Progress of Theoretical Physics Vol. 34, No. 2, pp. 204-209, (1965). DOI : 10.1143/PTP.34.204

[7] J. Kondo, "Giant Thermo-Electric Power of Dilute Magnetic Alloys." Progress of Theoretical Physics Vol. 34, No. 3,pp. 372-382, (1965). DOI : 10.1143/PTP.34.372

[8] J. Kondo,"Anomalous Density of States in Dilute Magnetic Alloys." Progress of Theoretical Physics Vol. 33, No. 4,pp. 575-584, (1965). DOI : 10.1143/PTP.33.575

[9] J. Kondo, "Review Resistance minimum and heavy fermions."Proc.JpnAcad Ser. BVol.82, p328,(2006). http://dx.doi.org/10.2183/pjab.82.328

[10] A.D. Brailsford, and A. W. Overhauser, "Theory of the Resistance Minimum in Dilute Paramagnetic Alloys."Phys. Rev. Lett. 3, 331-332, (1959).

DOI:10.1103/PhysRevLett.3.331

[11] P.W Anderson. "Localized Magnetic States in Metals."Phys. Rev.124,41,(1961).

DOI: http://dx.doi.org/10.1103/PhysRev.124.41

[12] B.R. Coles,"Low-temperature resistivity behaviour of molybdenum-iron, niobium-iron and palladium-iron alloys." Philosophical Magazine Vol. 8, issue 86, pp. 335-337, (1963). DOI: $10.1080 / 14786436308211131$

[13] B.R. Coles,B.V.B. Sarkissian, and R.H. Taylor,"The role of finite magnetic clusters in Au-Fe alloys near the percolation concentration."Philosophical Magazine Part B Vol. 37, issue 4, pp. 489-498,(1978). DOI: 10.1080/01418637808225792

[14] C. Rizzuto,"Formation of localized moments in metals: experimental bulk properties."Rep. Prog. Phys. 37, 147, (1974). DOI:10.1088/0034-4885/37/2/001

[15] J. Friedel,"On some electrical and magnetic properties of metallic solid solutions."Can. J. Phys. 34(12A): 11901211,(1956). DOI:10.1139/p56-134
[16] P.W. Anderson,'Localized Magnetic States in Metals."Phys. Rev. 124, 41, (1961).

DOI: http://dx.doi.org/10.1103/PhysRev.124.41

[17] Blandin, and J. Friedel,"Propriétés magnétiques des alliages dilués. Interactions magnétiques et antiferromagnétisme dans les alliages du type métal noble-métal de transition."J. Phys. Radium 20, 160-68,(1959).

DOI: $10.1051 /$ jphysrad:01959002002-3016000

[18] J. Ruvalds,"Resistance Minima in Magnetic Alloys."NATO Advanced Study Institutes Series Volume 81, pp 259-287, (1983). DOI:10.1007/978-1-4613-3497-2_7

[19] J.Souletie, "Relation Between the Anomalous Resistivity and the Susceptibility for Dilute Magnetic Alloys."Journal of Low Temperature Physics Vol. 7, No. 1/2, DOI: $10.1007 / \mathrm{BF} 00629125$

[20] L. MKovner," Theory of the Kondo effect at low temperatures."Zh. Eksp. Teor. Fiz. 65, 307-318,(1973). http://www.jetp.ac.ru/cgi-bin/dn/e_038_01_0151.pdf

[21] Yosuke. Nagaoka, "Self-consistent Treatment of Kondo's Effect in Dilute Alloys."Phys. Rev. B, 138, A1112A1120,(1965). DOI:10.1103/PhysRev.138.A1112

[22] M. A. Ruderman, and C. Kittel, "Indirect Exchange Coupling of Nuclear Magnetic Moments by Conduction Electrons. "phys. Rev.96, 99, (1954). DOI:10.1103/PhysRev.96.99; T. Kasuya,"A Theory of Metallic Ferro- and Antiferromagnetism on Zener's Model."Prog.Theor.Phys.16,45-57,(1956).

http://ptp.oxfordjournals.org/content/16/1/45; $\quad$ K Yosida, "Anomalous Electrical Resistivity and Magnetoresistance Due to an s-d Interaction in $\mathrm{Cu}-\mathrm{Mn}$ Alloys."Phys.Rev.107,396,(1957).

DOI:10.1103/PhysRev.106.893.

http://dx.doi.org/10.1103/PhysRev.107.396

[23] D. R. Hamann, "New Solution for Exchange Scattering in Dilute Alloys."Phys. Rev. B, 158, 570-580,(1967). DOI: 10.1103/PhysRev.158.570

[24] Zhu Bang- jieand Yao Kai-lun. "An application of new transport equation to low temperature resistivity of dilute alloys."Commun. in theor. Phys.(Beijing, China) vol.5,No.4,p.291, (1986). ISSN: 0253-6102CN: 11-2592/O3

[25] M. Zarea, S. E. Ulloa, and N.Sandler,"Enhancement of the Kondo effect through Rashba spin-orbit interactions."Phys. Rev. Lett.108, 046601,(2012). PMID: 22400870

[26] L.Isaev, D. F. Agterberg, and I. Vekhter, "Kondo effect in the presence of spin-orbit coupling.",Phys. Rev. B85, 081107,(2012). DOI:10.1103/PhysRevB.85.081107

[27] Alexander Cyril Hewson,"The Kondo Problem to Heavy Fermions." (Cambridge University Press, Cambridge, UK. (1993). http://dx.doi.org/10.1017/CBO9780511470752

[28] T. Yanagisawa,"Kondo Effect in the Presence of Spin-Orbit Coupling."J.Phys.Soc.Jpn.81, 094713, (2012). DOI:10.1143/JPSJ.81.094713

[29] Matteo Bazzanella and Johan Nilsson,"Ferromagnetism in the one-dimensional Kondo lattice: Mean-field approach via Majorana fermion canonical transformation." Phys. Rev. B 89, 035121,(2014). http://dx.doi.org/10.1103/PhysRevB.89.035121 
[30] Jie Wang andDimitrie Culcer."Suppression of the Kondo Resistivity Minimum in Magnetic Topological Insulators." Phys.Rev.B88,125140,(2013).

DOI: $10.1103 /$ PhysRevB.88.125140

[31] M.A.AL-Jalal"Phenomenological analysis of the s-d exchange interaction in dilute $\mathrm{Cu}-\mathrm{Mn}$ alloy at helium temperature."Adv.Mat.Lett.,5(1),(2014).

DOI: $10.5185 /$ amlett.2013.5486

[32] M. Ramanzani, H.Sauer,A M Holz,.R. Birringer, and H. Gleiter,"On the Kondo-effect in dilute alloys of iron and nanoscaled copper."Zeitschrift für Physik B Condensed Matter Volume 100, Issue 1, pp 39-45, (1996). DOI: $10.1007 / \mathrm{s} 002570050091$

[33] F. W. Smith "Strength of the Ruderman-Kittel-Kasuya-Yosida interaction in dilute AgMn alloys."Phys. Rev.B, 13, 2976 , (1976). DOI: http://dx.doi.org/10.1103/PhysRevB.13.2976
[34] D. A. Smith,"The zero-frequency susceptibility of spin glasses and mictomagnets." J. Phys. F: Metal Phys. 5, 2148,(1975). DOI: $10.1088 / 0305-4608 / 5 / 11 / 027$

[35] A. Kaucha andK. Byczuk, "Variational local moment approach: From Kondo effect to Mott transition in correlated electron."Physica B: Condensed Matter. Volume 407, Issue 2, Pages209-217,(2012).

http://dx.doi.org/10.1016/j.physb.2011.10.030

[36] K. H. Fischer,"Kondo effect in spin glasses: Electrical resistivity."Zeitschriftfür Physik B Condensed Matter 42(1):27-38, (1981). DOI:10.1007/BF01298289

[37] Y. C. Tsayand L. Shen, "The electrical resistivity of dilute magnetic alloys: Kondo effect vs spin glass behavior."J. Applied Physics; 49, 1625-1626. (1978). DOI:10.1063/1.32492. 